Article

\title{
A Computational Fluid Dynamics Model for a Water Vortex Power Plant as Platform for Etho- and Ecohydraulic Research
}

\author{
Dennis Powalla $^{1}{ }^{\circledR}$, Stefan Hoerner ${ }^{1, *}\left(\mathbb{D}\right.$, Olivier Cleynen ${ }^{1}\left(\mathbb{D}\right.$, Nadine Müller $^{2}$ and Jürgen Stamm ${ }^{2}$ \\ and Dominique Thévenin ${ }^{1}$ (D) \\ 1 Laboratory of Fluid Dynamics and Technical Flows, University “Otto von Guericke" of Magdeburg, \\ Universitätsplatz 2, 39106 Magdeburg, Germany; dennis.powalla@ovgu.de (D.P.); \\ olivier.cleynen@ovgu.de (O.C.); dominique.thevenin@ovgu.de (D.T.) \\ 2 Institute of Hydraulic Engineering and Technical Hydromechanics, TU Dresden, 01062 Dresden, Germany; \\ nadine.mueller@wsv.bund.de (N.M.); juergen.stamm@tu-dresden.de (J.S.) \\ * Correspondence: hoerner@ovgu.de
}

check for updates

Citation: Powalla, D.; Hoerner, S.; Cleynen, O.; Müller, N.; Stamm, J.; Thévenin, D. A Computational Fluid Dynamics Model for a Water Vortex Power Plant as Platform for Etho- and Ecohydraulic Research. Energies 2021, 14, 639. https://doi.org/10.3390/ en14030639

Academic Editor: Paul Christodoulides Received: 10 December 2020 Accepted: 23 January 2021 Published: 27 January 2021

Publisher's Note: MDPI stays neutral with regard to jurisdictional clai$\mathrm{ms}$ in published maps and institutional affiliations.

Copyright: (C) 2021 by the authors. Licensee MDPI, Basel, Switzerland. This article is an open access article distributed under the terms and conditions of the Creative Commons Attribution (CC BY) license (https:// creativecommons.org/licenses/by/ $4.0 /)$.

\begin{abstract}
The objective of the present paper is to develop a validated numerical model of a water vortex power plant that serves as a digital twin for further studies such as assessments of the ethohydraulic characteristics or the performance of such devices. The reference for the validation process is a large-scale hydraulic installation equipped with a full-scale water vortex power plant prototype installed in Dresden (Germany), where flow field measurements were carried out using three-dimensional Acoustic Doppler Velocimetry. The numerical model was implemented within the software package Star-CCM+. The unsteady, two-phase flow was solved with the Reynolds-Averaged Navier-Stokes equations in a Eulerian Multiphase approach, deploying a Volume of Fluid method to describe the free-surface flow. Water level and flow velocities were systematically compared in key areas of the device, demonstrating that the simulation is in good agreement with experimental observations. Relative differences are limited to at most $4 \%$ regarding water height in the system, and even the much more challenging velocity fields are reproduced with typical relative errors of roughly $10 \%$. This validates the ability of the model to model the challenging flow conditions found in a water vortex power plant, enabling subsequent studies of the characteristics of this power plant concerning fish migration.
\end{abstract}

Keywords: water vortex power plant; computational fluid dynamics (CFD); Reynolds-Averaged Navier-Stokes (RANS); eulerian multiphase; volume of fluid (VOF)

\section{Introduction}

Energy consumption increases along with the world population, but also due to higher energy consumption per capita compared to former generations. Most of the energy is currently provided by fossil resources. The resulting climate change and environmental pollution have therefore serious consequences for the ecological system [1]. To address these negative effects, sustainable and environmentally friendly energy systems have emerged and are politically promoted over the last decades, aiming for alternatives to existing fossil energy resources. The challenge is a transition towards carbon free, sustainable and environmentally friendly energy systems.

One pillar of renewable energy exploitation technologies is hydropower. Hydraulic power plants can be classified by different categories such as type of construction, their turbines and by the form of energy they extract. The two main energy forms are potential energy in storage power plants and kinetic energy in river power plants [2]. Small-scale, low-head power plants are of particular interest for off-grid applications or deployment in regions where access to electricity is not always guaranteed [3]. However, they often strongly impact the surrounding ecosystems, in particular regarding those devices which block the natural continuity of a river. This is typically the case of run-of-river power plants, 
where a dam interrupts fish passage in both directions, compromising the sustainability of fish populations [4]. In Europe, run-of-river power plant operators have to conform to the European Water framework directive, which states the requirements related to their ecological impact [5]. To enable fish passage, the current state of the art consists of fish ladders or lifts, which offer alternative paths for fish and reconnect the upper and lower parts of the river separated by the dam. In Germany, the design of such devices is regulated by the directive of the German Association for Water [6]. These devices are expensive [7], and some do not perform as intended [8-10]. The research network "Wachstumskern Flussstrom Plus" aims to develop hydraulic power with low ecological impact thanks to innovative strategies.

One of the devices developed as part of this network is the so-called "Fish-Friendly Weir" (FFW). The FFW is a water vortex power plant (WVPP), which was originally designed by the company Ecoligent and is intended for implementation as an alternative to fish ladders. The probed plant is conceived for a head of $1 \mathrm{~m}$. A cascade installation (with several weirs installed in series) would be suitable for higher heads. The basic concept of a WVPP is to lead incoming water tangentially into a vortex chamber, leaving through a central orifice at the bottom. The geometry of the chamber forces the fluid to develop a vortex without any guide vane. The fluid's rotational energy is converted into work by a turbine with a vertical axis, and ultimately transferred to a generator for electrical production. Various variants of the FFW have already been investigated with a focus on fish compatibility $[11,12]$, but without extensive experimental validation. Other experimental studies focused on the hydrology, but the dimensions of these devices were only at laboratory scale [13-16].

By contrast, the installation of a full-scale prototype at the Technical University of Dresden (TUD) allows for a systematic scientific study with realistic conditions [17]. Experimental investigation were carried out, assessing fish compatibility using tests deploying live fish under laboratory conditions [18].

The authors already carried out research into the ethohydraulics and fish passage quality of a similar device in the past [11,12]. Now, the availability of full-scale experimental data in Dresden opens the door for further research using numerical tools, systematically investigating the etohydraulics and fish passage quality of the device using computational fluid dynamics (CFD). However, a necessary first step for this is the setup of a suitablycalibrated and validated numerical model.

Despite a relative simple geometry, the numerical investigation of a WVPP is challenging due to the complex free surface generated by the vortex and, thus, its flow field in the vortex chamber. The numerical models of a free surface water vortex provide the basis for the representation of a WVPP in a CFD-framework. One approach using a Finite Volume Method (FVM) is to represent the phases (in the present case air and water) by a Eulerian-multiphase flow model. In this paper, a simplified approach is deployed, using a single Eulerian scheme that solves the entire flow, while the interface of the two phases is captured by volume-of-fluid (VOF) method, as shown by Mulligan [19]. Adding a rotating device (turbine), which interacts with the flow, increases the complexity even further. Then, besides the capturing of the interface of the two phases, the model has to maintain an information transfer from a static region to an embedded rotating region as well. This often results in stability issues and leads to high requirements regarding mesh quality. This is especially true in the study at hand featuring high velocity gradients, typical for rotating parts in the vicinity of the wall. Additionally, up to now, WVPPs are not extensively researched with numerical means. Mueller [11] addressed the aforementioned challenges and solved it by geometrical simplifications allowing for the set up of a numerical stable model. It has to be noted that the scale of their model is based on the dimensions of an existing plant, but the results could not be validated due to a lack of reliable measurement data. The WVPP is described by 3D simulations using a hybrid-RANS-LES model. While the scope in this study was about downstream fish migration, the numerical part was not described extensively. Nishi and Inagaki [20] validated their numerical model in a small-scaled laboratory set up with reduced physics. Their focus was mainly set on the performance characteristics of the runner. 
Dhakal et al. (2017) performed a series of studies on vortex power plants [21-23]. To this, they used a configuration of a WVPP much different to the geometry discussed in here. The basin of the vortex chamber is conical shaped, which focuses on an increased power output rather than fish compatibility. Despite the complex, dynamical multiphase flow in a GWVPP, the numerical simulations were carried by a single phase, steady-state model, in a closed domain, whereas the laboratory set up is a free surface installation.

The objective of this paper is to develop a three-dimensional unsteady turbulent CFD model of the installation, as well as the validation of the simulations against experimental measurements. The focus is set on fluid flow properties, such as water level and velocity fields in predefined areas. If successful, this model will serve as a base for further studies on WVPP, ultimately providing deeper knowledge of its hydraulic and ecological characteristics. It will ultimately be possible to assess its performance regarding fish migration using computer simulations. The numerical model introduces a digital twin with reasonable computational costs for a full scale functional water vortex power plant featuring a high complex multiphase flow. It is intended as a robust and reliable platform for subsequent numerical investigations with different scope such as turbomachinery, fluid dynamics and eco- and ethohydraulic studies. Especially ethohydraulic researchers in our team will benefit from our approach, providing a thorough three-dimensional information of the time-resolved flow field which is mostly not available. The detailed description of the numerical model and setup will allow for a simple reproduction of set up applied and serve as a template for further research on WVPP.

\section{Materials and Methods}

\subsection{Numerical Setup}

The numerical model was built with the use of the Star-CCM+ software package. The two-phase flow was described by a Eulerian Multiphase Model and the volume-offluid (VOF) method [24]. The VOF method is a simple multiphase flow model capable of resolving the interface between the phases of the mixture by adding a volume fraction transport equation. For two phases, only one volume transport fraction is calculated. It is assumed that no interface interaction occurs, and therefore all phases share the same velocity, pressure and temperature fields. It is an efficient method but less accurate than a Eulerian-Eulerian method, where momentum and continuity equations for each phase are solved. A segregated SIMPLE (Semi-implicit Method for Pressure Linked Equations) solver was chosen. Reynolds-Averaged Navier-Stokes (RANS) equations were deployed to capture the highly turbulent, fluctuating flow with reasonable computational costs. More sophisticated, but also much more expensive, methods, e.g., Large-Eddy Simulations (LES), were not considered, since many operating points of the device shall be investigated in future work. Based on preliminary studies, the two-equation SST (shear stress transport) $k-\omega$ approach introduced by Menter was chosen for turbulence modeling [25], where $k$ is the turbulent kinetic energy and $\omega$ is the specific rate of dissipation. As turbulence is anisotropic, these models under predict anisotropy, which affects the flow field [26]. Considering the trade-off in between numerical robustness and computational costs with the accuracy of the model and the validation results, the approach was estimated to be sufficient for the task. The boundary layer was captured by an adaptive $y^{+}$wall treatment, where $y^{+}$is the non-dimensional wall distance. A mass flow inlet boundary condition was set at the inflow, since the mass flow is known from the experimental investigations [27]: the solver then produces a uniform mass flux on each face of the boundary. Where the open device interacts with the environment (atmosphere and domain outlet), a pressure outlet boundary condition is set. This boundary pressure can be considered as the static pressure of the environment (here, static pressure of air and water) into which the fluid enters. The boundary face velocity is extrapolated from the inner domain and the static pressure. The outlet water height level is controlled by a step, which represents the weir used at a the actual device. The walls bounding the domain and the turbine surfaces 
were set to a no-slip condition. Figure 1a displays the domain, where different types of boundaries are highlighted in different colors.

(a)

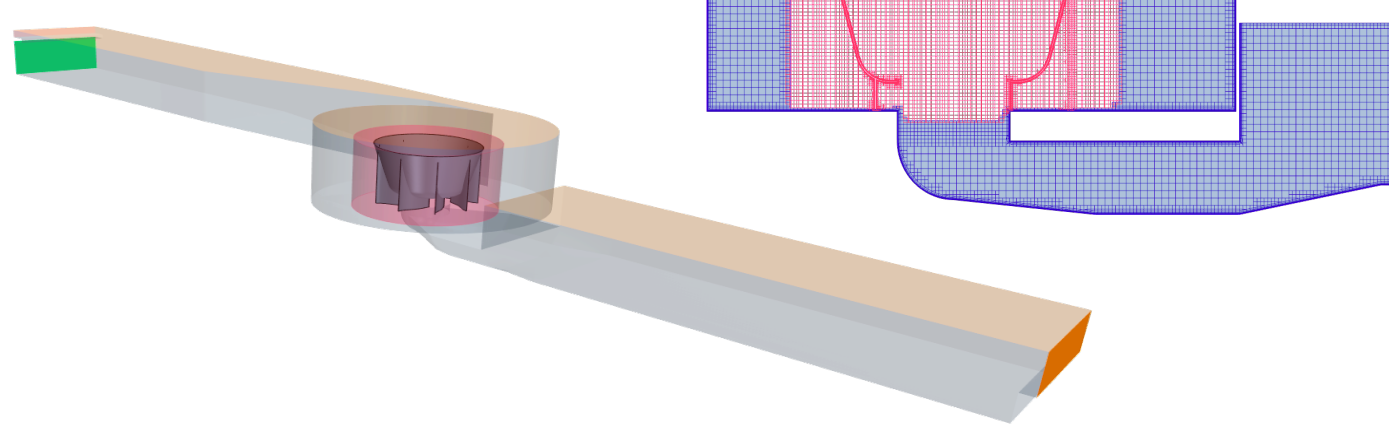

Figure 1. (a) The boundary types in the numerical set up: mass flow inlet boundary (green), pressure outlet boundary (light and dark orange), overset region (red cylinder containing turbine geometry) and wall/no-slip boundary condition (light grey). (b) The mesh resolution at the Vortex chamber. The main cell size in the background region (blue) is $0.05 \mathrm{~m}$, and it is $0.025 \mathrm{~m}$ in the refined area/overset region (red). The data transfer between both meshes occurs where both overlap.

The turbine motion was implemented using an overset mesh approach, which discretizes the computational domain with two different meshes (background mesh and moving overset mesh) that overlap (Figure $1 \mathrm{~b}$ ). The gap between rotor blades and chamber floor is only about $1 \mathrm{~cm}$, which translates into high velocity gradients and represents a challenge for numerical stability. The spatial discretization is made of hexahedral cells with trimmed cells refining the mesh in the vicinity to the boundaries. To properly capture viscous gradients next to wall boundaries, prismatic cell layers were added. The full geometry is finally represented by 4.7 million cells. The temporal discretization of the transient term is of first order and the time step is set to $5 \mathrm{~ms}$. The mesh representing the turbine device rotates at a speed of $27 \mathrm{rpm}$, as does the turbine in the actual device. An overview of the simulation set up is shown in Table 1.

The flow field in a WVPP is highly unsteady in several regions. In the vortex basin, free surface effects and the interaction between the main vortex and the turbine dominate the dynamics. The range of the Reynolds numbers in the domain is found to be in between $1.5 * 10^{6}$ and $6.2 * 10^{6}$. Lowest Reynolds number are found for the upstream and downstream channel, while highest turbulence is given in the duct geometry. Due to the geometry, the turbine outlet features an unsteady vortex, which dissipates into a highly-turbulent wake that settles into the outlet channel. Another peculiarity of this hydraulic system is the interplay of mass flow and water level at inlet and outlet. In the simulation, a suitable balance of the resulting over-determined system was successfully realized by iterative adjustment of the outlet pressure condition.

All simulations shown in what follows were run in parallel on 5 nodes (16 coreHaswell), corresponding to 80 CPUs on the high-performance Linux cluster Neumann of the University of Magdeburg, progressing at a rate of $0.62 \mathrm{CPU}$ hours per simulated second of physical time. One CFD simulation requires approximately 19,200 CPU-hours in total, which corresponds to five days. 
Table 1. Overview of numerical set up.

\begin{tabular}{|c|c|}
\hline Solver physics & 3d, implicit unsteady, turbulent, Eulerian-multiphase, segregated flow \\
\hline Solver algorithm & SIMPLE \\
\hline \multicolumn{2}{|l|}{ Temporal discretization } \\
\hline Accuracy & first order \\
\hline Time step & $5 \times 10^{-3} \mathrm{~s}$ \\
\hline \multicolumn{2}{|l|}{ Spatial discretization } \\
\hline mesh type & core region: trimmed mesh (hexahedral cells), \\
\hline & near wall region: Prism layer mesh (orthogonal prismatic cells) \\
\hline core cell size: Background mesh & $0.05 \mathrm{~m}$ \\
\hline core cell size: Overset mesh & $0.025 \mathrm{~m}$ \\
\hline Total amount of cells & $\approx 4.7$ million \\
\hline Turbulence model & (Menter) SST $k-\omega$ \\
\hline Wall treatment model & All $y^{+}$wall treatment \\
\hline Multiphase model & Volume of fluid (VOF) \\
\hline \multicolumn{2}{|l|}{ Convergence criteria } \\
\hline Continuity equation & residual target $=10^{-4}$ \\
\hline $\mathrm{X}-, \mathrm{Y}-$ and $\mathrm{Z}$ momentum equation & residual target $=10^{-4}$ \\
\hline Calculation effort & $\approx 19,200 \mathrm{CPU}$ hours \\
\hline
\end{tabular}

\subsection{Experimental Setup}

The full-scale model of the WVPP installed at TU Dresden (see Figure 2) was built to perform fluid flow measurements and to assess the fish migration qualities of the prototype [27]. Flow velocities and water levels were measured by the project partner team at TUD, in order to assess the device's compliance with ecological regulations. These data serve as the reference for the study at hand.

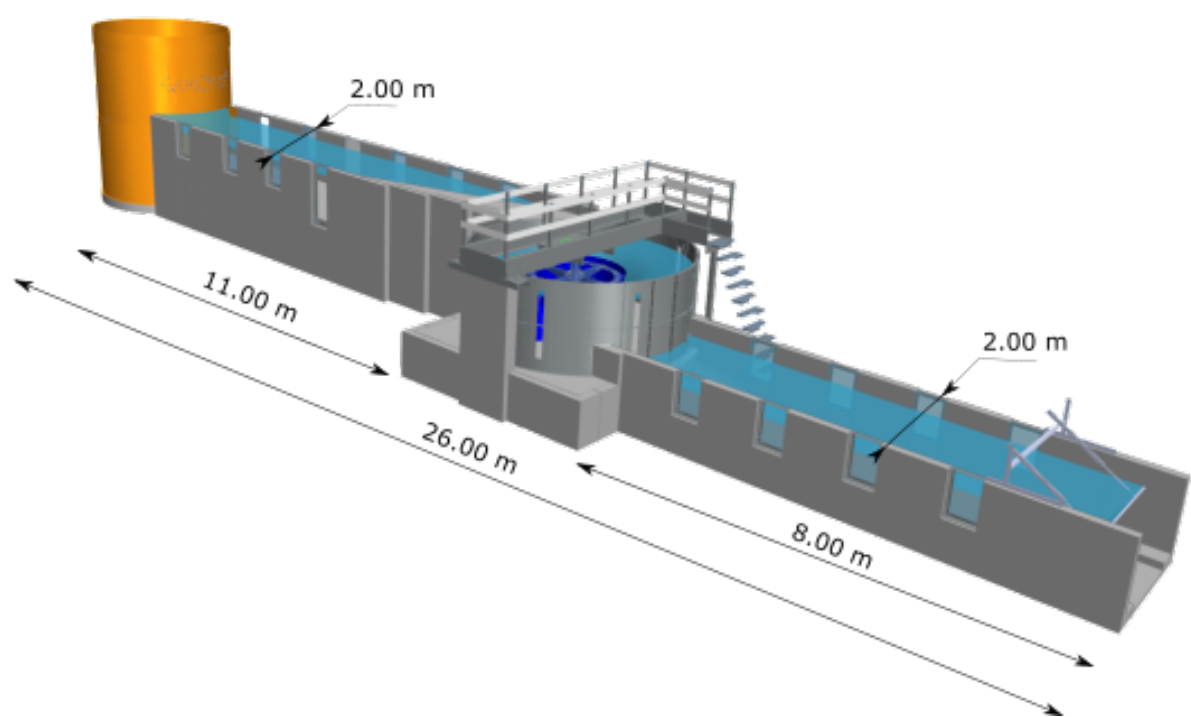

Figure 2. Full-scale WVPP model at TUD [28]. The total length of the installation is about $26 \mathrm{~m}$; the upstream channel is $11 \mathrm{~m}$ long and the downstream channel is $8 \mathrm{~m}$ long. The spiral-shaped vortex chamber is based on two radii, where the larger radius is $1.98 \mathrm{~m}$ and the smaller one is $1.68 \mathrm{~m}$. The diameter of the orifice at the center of the vortex chamber is $0.80 \mathrm{~m}$. The integrated turbine has a height of $1.00 \mathrm{~m}$ and a diameter of $1.75 \mathrm{~m}$ and consists of a dome with nine attached blades radial equally distributed. The geometry of this installation was provided by the industrial research partner Ecoligent. 
Acoustic Doppler Velocimetry (ADV) was deployed by TUD researchers for the velocity measurements. These probes provide a three dimensional velocity vector of the flow in a sampling volume of less than $1 \mathrm{~cm}^{3}$. The sensor was fixed to a linear drive system, allowing for point measurements at different positions and in different cross-sections of the device. The flow velocity was captured at the vortex chamber inlet and in the vortex chamber itself. The velocity values for each measurement point were averaged over approximately $300 \mathrm{~s}$ at a rate of 25 samples per second. Furthermore, the water levels were probed by ultrasonic sensors at the inlet and outlet and in the vortex chamber, as shown in Figure 3. These sensors measured the water level transversal to main flow. Their averaged value were taken over a period of $150 \mathrm{~s}$ at a rate of 1 sample per second. In this experiment, the operating conditions were set to the best efficiency point of the device used in the design process by the developer (Ecoligent). Thus, the volume flow rate was $0.7 \mathrm{~m}^{3} \mathrm{~s}^{-1}$, the rotational speed of the turbine was set to $27 \mathrm{rpm}$ and the water height drop between the inlet and the outlet channel was set to $\Delta h=1 \mathrm{~m}$.
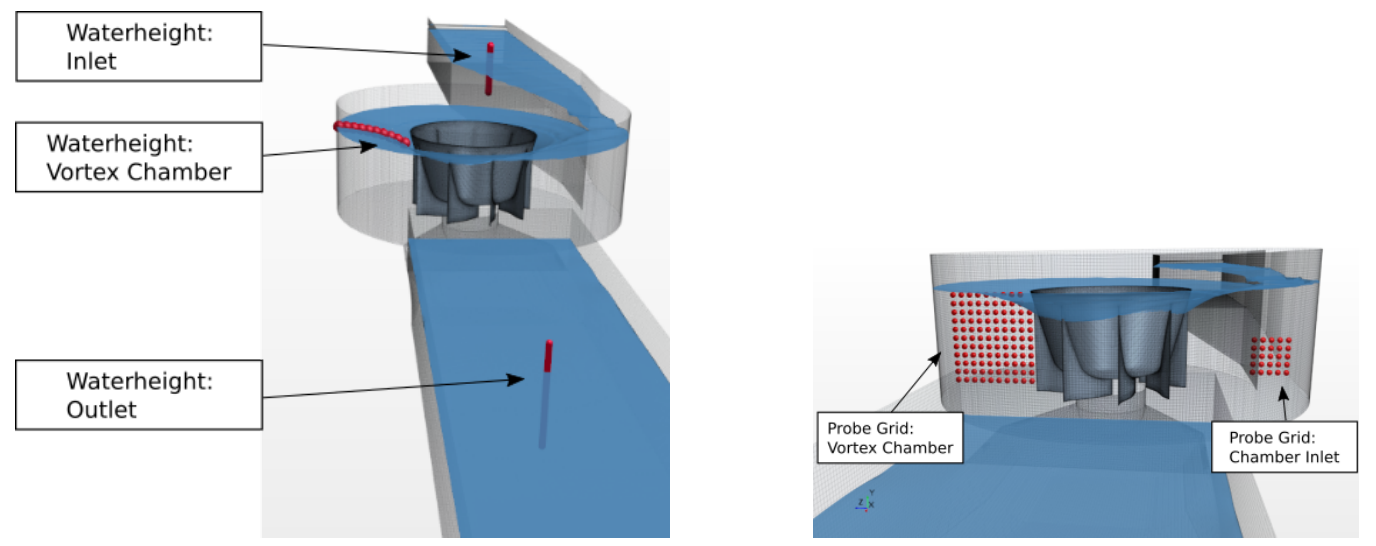

Figure 3. Three-dimensional model of the weir showing the position of the water level and velocity probes. (Left) Probes for the water level at inlet and outlet and in the vortex chamber. In the vortex chamber, probes were taken at a distance of $0.1 \mathrm{~m}$ from the wall towards the center until reaching the tip of the turbine blades. (Right) The distance in between the grid points used for velocity measurements is $0.1 \mathrm{~m}$. Thus, the resulting measurement grids for the velocity probes are matrices with the size $5 \times 5$ at the vortex chamber inlet and $10 \times 11$ in the vortex chamber near the turbine.

\subsection{Mesh Independence}

A grid convergence study was first carried out to ensure that the obtained solution did not depend on the mesh resolution. For this purpose, four meshes of different refinement levels were used, using the mesh with the finest resolution as a reference. Since the velocity field in the vortex chamber is of high interest for this study, a representative selection of probe points was chosen among the available experimental data. The velocity values at those points were averaged after a quasi-steady state was reached in the simulation; they are plotted in Figure 4. Convergence was considered as satisfactory when the residues for the three momentum and the continuity equation are below $10^{-4}$. As additional stopping criteria, the mass flow rate for inlet and outlet, the water height at inlet and outlet and the moment acting on the turbine were selected. All these criteria are linked via a logical AND and are considered satisfied when their standard deviation does not exceed $10 \%$ over a period of 500 consecutive time steps, indicating that stable values have been found. After reaching quasi-steady state, the simulation was finally pursued during $10 \mathrm{~s}$ of physical time (4.5 rotations of the turbine), and the results were averaged during this lapse of time. Naturally, a finer mesh is associated to higher computational costs. Therefore, the computation time per time step is also plotted in Figure 4. 


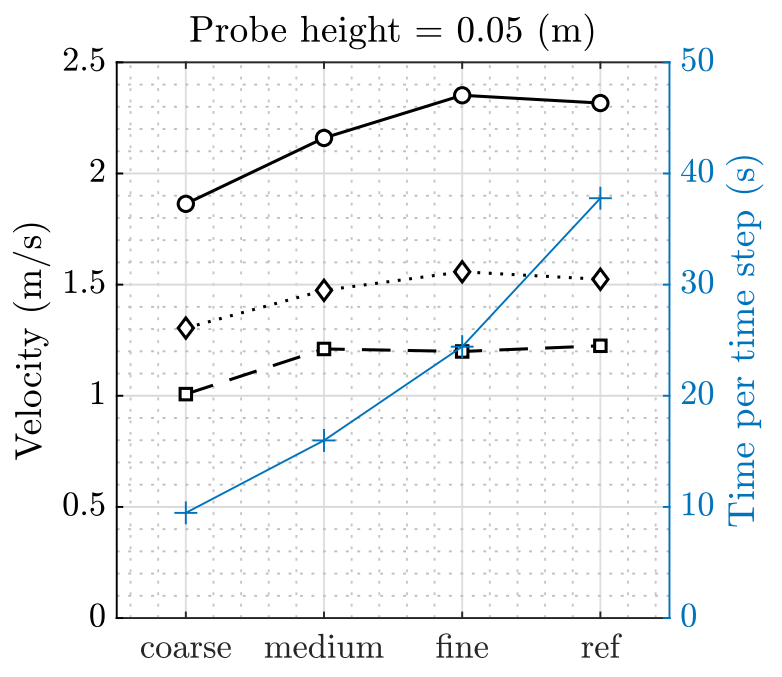

\begin{tabular}{|c|}
\hline Radius $(\mathrm{m})$ \\
\hline 一 ㅁ -1.85 \\
$\cdots$
\end{tabular}

(a)

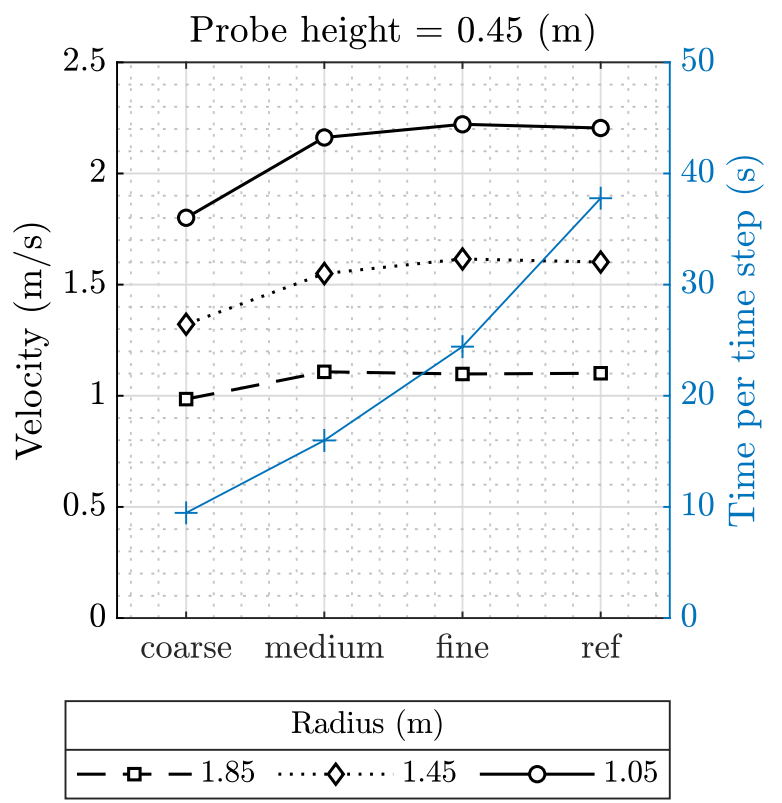

(b)

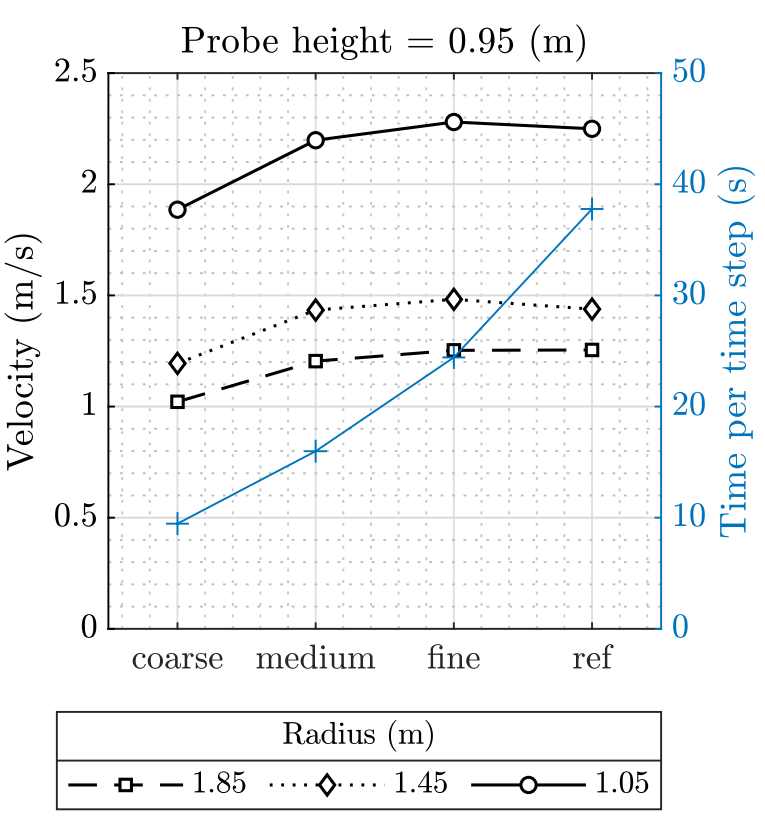

(c)

Figure 4. (a-c) The averaged velocity obtained by CFD for different mesh refinement levels (from left to right: from coarse to finest with mesh size of $0.05,0.03,0.02$ and $0.015 \mathrm{~m}$ ) at different locations within the vortex chamber. Each plot corresponds to a different water height from bottom and shows measurements at three different radii (from bottom to top: close to chamber wall, in the core and close to turbine blades). The blue line shows the computational time per time step using the right-hand vertical axis.

The trends shown in Figure 4 are identical for all probe points, as confirmed by the results in Table 2. Considering the large fluctuations found in this turbulent flow (see next) and taking into account the uncertainty associated to process conditions and measurement systems, a relative accuracy of 5\% was considered a suitable target for the numerical model. The analysis of these results reveals that this accuracy is obtained for all quantities with the medium refinement level (i.e., a mesh with 4.7 million cells). As a consequence, this mesh was retained for all further studies. 
Table 2. Averaged values for velocity (vel.-av) in $\mathrm{m} / \mathrm{s}$ are taken exemplarily for the point at height $0.45 \mathrm{~m}$ and radius $1.05 \mathrm{~m}$. In the right column, the corresponding deviation (dev.) compared to the reference value (on the finest mesh) is shown. Mesh number goes from coarse (1) to reference (4).

\begin{tabular}{cccc}
\hline Mesh & Cells & vel.-av. & dev. (\%) \\
\hline 1 & $2,083,132$ & 1.895 & 10.3 \\
2 & $4,579,779$ & 2.169 & 3.62 \\
3 & $7,146,041$ & 2.283 & 0.11 \\
4 & $11,734,809$ & 2.251 & ref \\
\hline
\end{tabular}

In the vortex chamber, strong velocity fluctuations are observed in the laboratory model as well as in the numerical model as a consequence of the interaction between turbine rotation and free-surface effects. The impact of mesh refinement on the fluctuations is displayed in Figure 5, at the highest probe point closest to the turbine blades. These results confirm that the medium refinement level displays suitable results.

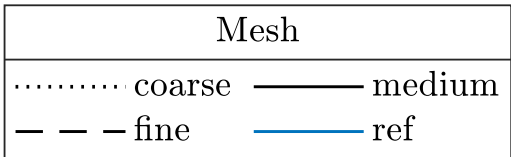

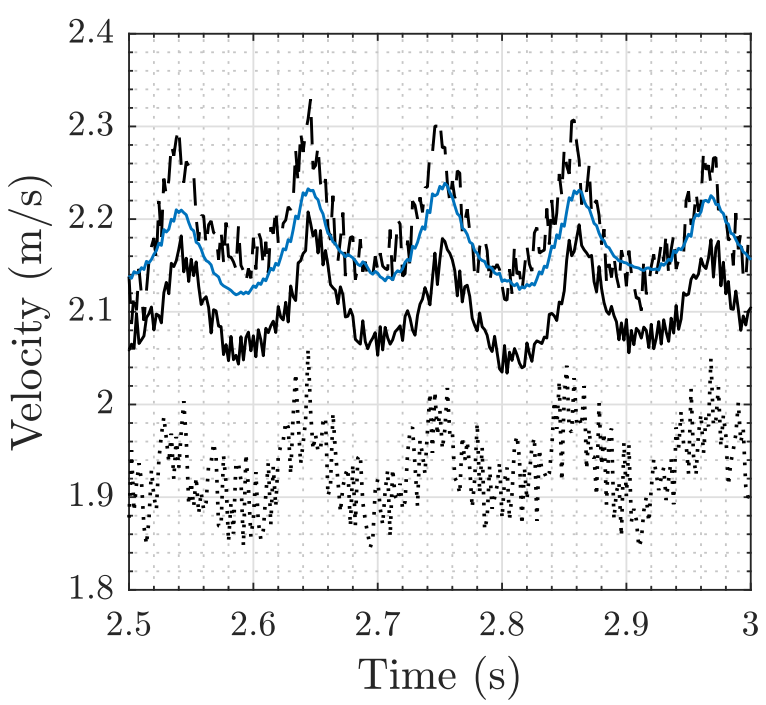

(a) Velocity fluctuations over time (time was set to zero after reaching quasi-steady state, corresponding here to about 110 s of physical time)

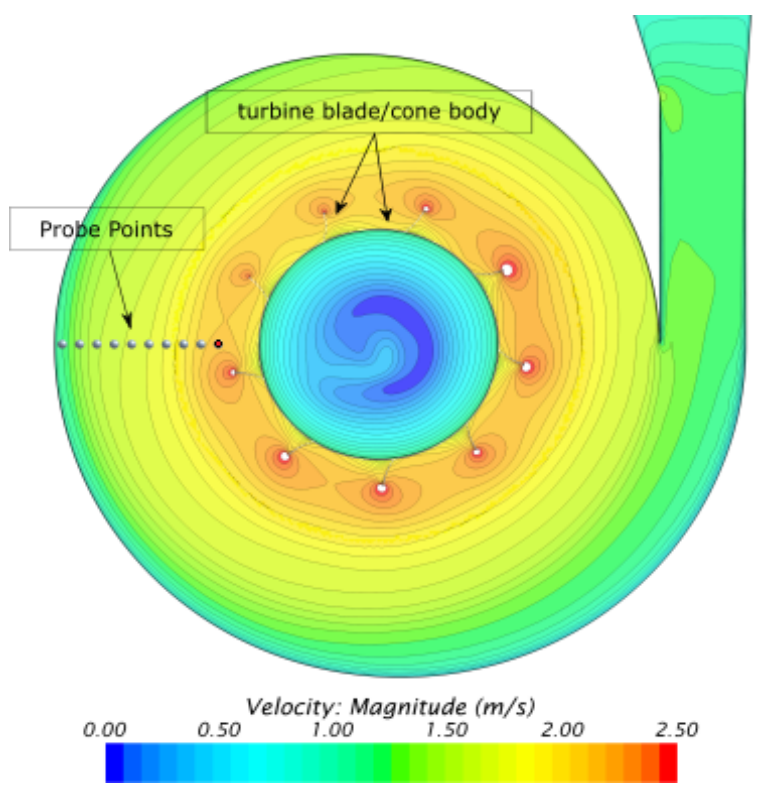

(b) Top view showing instantaneous velocity field at $0.45 \mathrm{~m}$ height from vortex chamber bottom.

Figure 5. (a) Velocity fluctuations for four different level of mesh resolution, where blue is the reference mesh (seen in (a)). The velocity probe is located mid-height in the chamber and nearest to the turbine. (b) A cross plane section displaying the velocity field through the vortex chamber, at the height of the mentioned velocity probe (red highlighted point seen in (b)).

\section{Results}

The water levels in the simulation and experiment are shown in Figure 6. The height at each probe point is plotted over the radius of the vortex chamber, from the vicinity of the turbine blades (on the left, lowest water level) to the external wall of the vortex chamber (on the right, highest water level).

Both curves in Figure 6 capture the water surface altitude increase as the radius is increased toward the external wall. This is a general characteristic of the development of a water-surface vortex. The curves of the experiment and the simulations show overall a very good agreement. However, small relative differences, increasing towards the center of the vortex chamber, are found. The maximum difference is found at the point closest to the turbine impeller, which is also the point indicating the highest variations (and hence 
the highest uncertainty) in the experiments. However, the mean percentage deviation over all probes is merely $2.5 \%$. Therefore, it can be concluded that the water level in the vortex chamber is well captured by the CFD simulation.

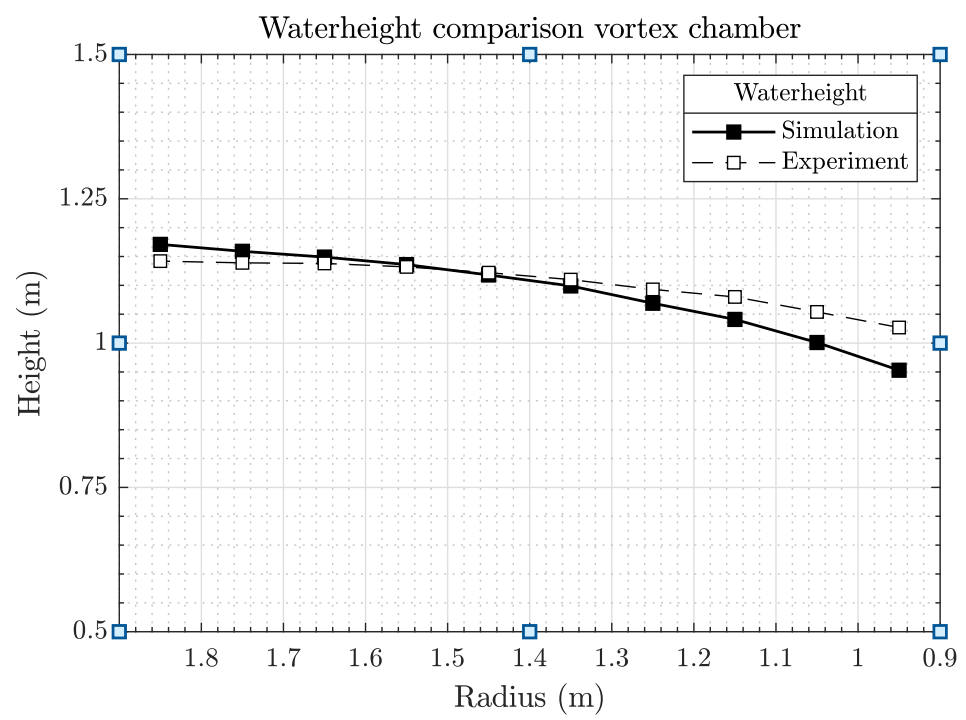

Figure 6. Water level comparison between simulation and experiment in the vortex chamber. The outer wall is situated at a radius of $1.95 \mathrm{~m}$ (on the right), while the turbine blades stop at a radius of $0.875 \mathrm{~m}$ (on the left). The mean deviation between numerical simulations and experimental measurements is $2.50 \%$ and the maximum deviation is $7.21 \%$.

A comparison between experimental measurements and numerical predictions of the water height at inlet and outlet, as well as the difference between them (the height drop across the device), is shown in Table 3. The water height at inlet and outlet are in very good accordance in the simulation and experiment, with deviations below $4 \%$. Even the head drop between inlet and outlet is well reproduced with only $5.4 \%$ relative difference, indicating a proper numerical description of the complex flow through the rotating turbine.

Table 3. Comparison of averaged values for water height at inlet and at outlet and water drop between inlet and outlet, together with the corresponding deviations (considering the experimental data as reference).

\begin{tabular}{cccc}
\hline & Experiments & Simulations & Relative Deviations in \% \\
\hline Water height at inlet $[\mathrm{m}]$ & 0.81 & 0.83 & 2.5 \\
Water height at outlet $[\mathrm{m}]$ & 0.75 & 0.72 & 4.0 \\
Drop between inlet and outlet [m] & 1.10 & 1.04 & 5.4 \\
\hline
\end{tabular}

Subsequently, a comparison between the velocity measurements and the calculated velocity fields was carried out. Exemplary instantaneous velocity fields obtained by CFD are shown in Figure 7, together with the locations of the experimental measurements (red dots). A comparison of the velocities at the chamber inlet is plotted as a function of the measurement position in Figure 8. For a better visualization, the measurement grid is divided into horizontal layers, and one plot is shown for each height. It can be seen that all curves are relatively flat, fit quite well, but show larger differences in the vicinity of the turbine. Flat curves indicate a homogeneous flow, which is expected in this region, located near the wall. The numerical predictions indicate almost perfect homogeneity, and they do not reveal slightly higher velocities near the inner wall, as observed in the experiments. However, the averaged difference between numerical and experimental results concerning the velocity magnitude in this region is only $7.5 \%$, which is still satisfactory. 


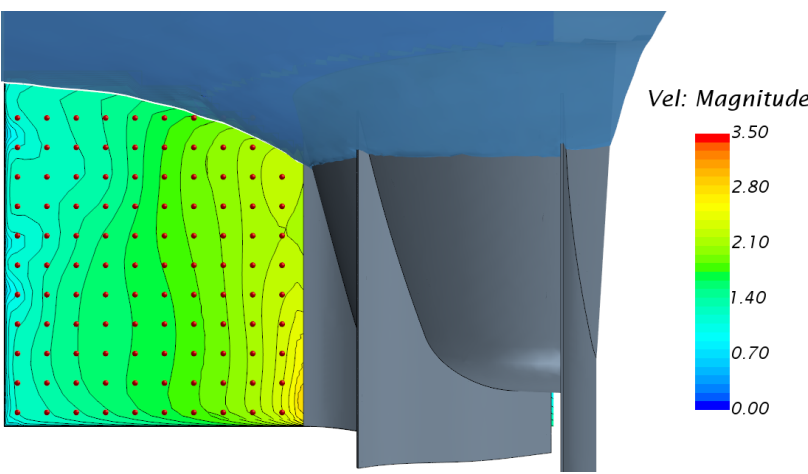

(a) Velocity field in vortex chamber

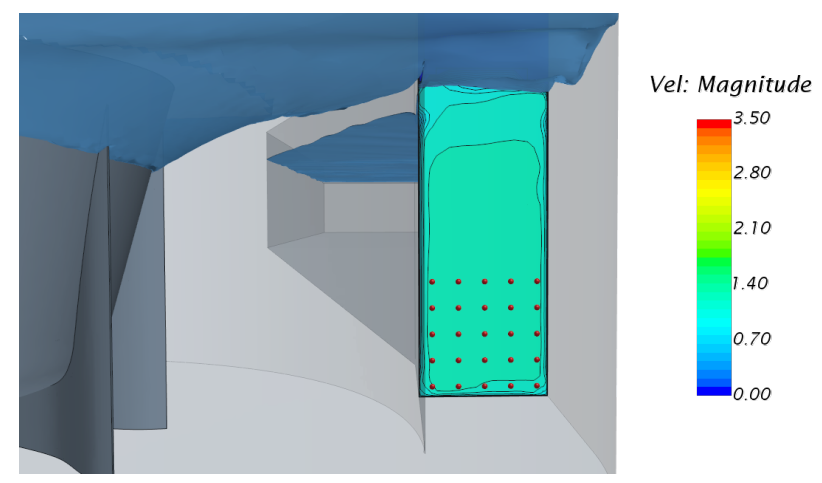

(b) Velocity field at chamber inlet

Figure 7. Exemplary instantaneous velocity fields obtained by CFD (with values in $\mathrm{m} \mathrm{s}^{-1}$ ), at both cross-sections where the experimental measurements were carried out. The red dots in both images show the positions where experimental velocity measurements are available. The water surface is shown in dark blue.
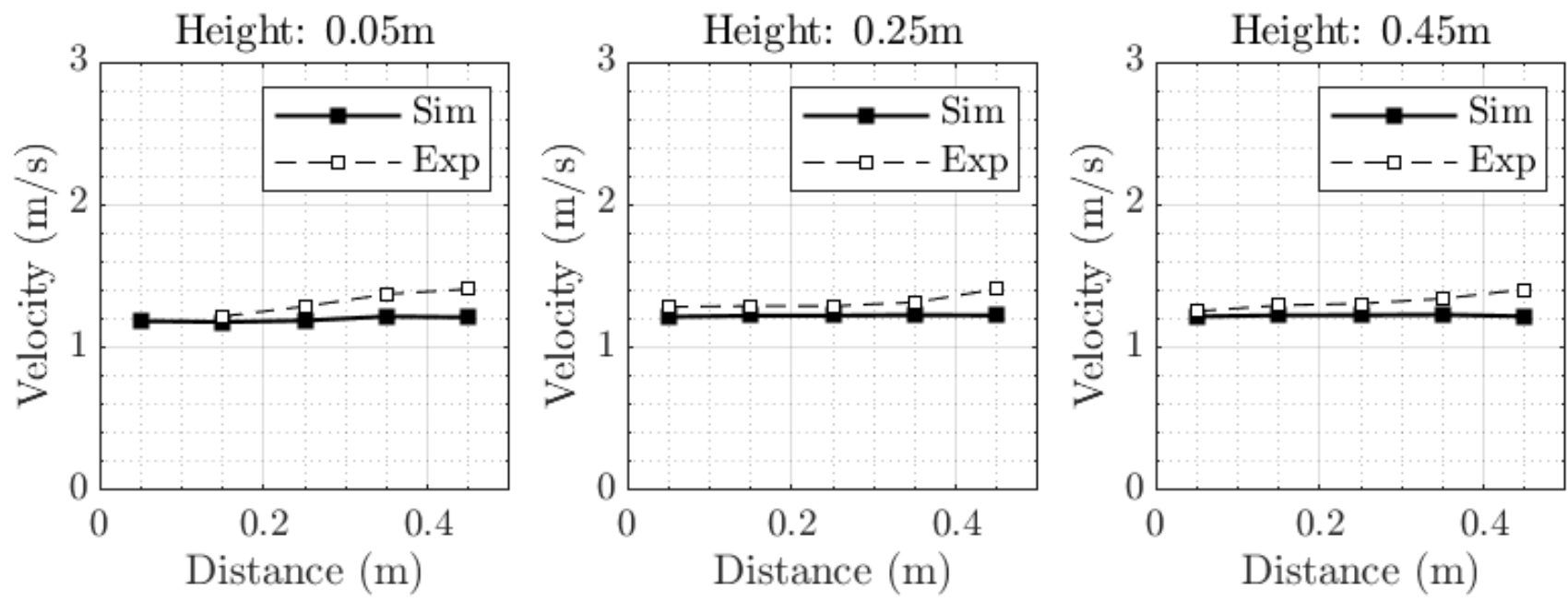

Figure 8. Comparison of velocity magnitude between simulation and experimental data at chamber inlet. The horizontal axis shows the horizontal position within the chamber inlet, where $0.00 \mathrm{~m}$ is the inner wall and $0.50 \mathrm{~m}$ is the outer wall. By reference to the measurement grid matrix shown in Figure 7, the results are shown at three different heights from bottom (lowest measurement line at $0.05 \mathrm{~m}$ (left); central measurement line at $0.25 \mathrm{~m}$ (middle); and top measurement line at $0.45 \mathrm{~m}$ (right)). The average relative difference between numerical and experimental results is, from left to right: $7.90 \%, 7.20 \%$ and $7.30 \%$. The maximum relative differences are: $11.35 \%, 10.12 \%$ and $12.91 \%$.

The same comparison was finally carried out for the vortex chamber itself, as shown in Figure 9. The curves agree very well qualitatively and show an increase of the velocity towards the center of the chamber (i.e., the turbine impeller, place on the left side of the graphs). In general, it can be seen that the simulation mostly overestimates slightly the velocities compared to the experimental measurements. Taking all measurement points into account, the mean deviation between experiments and simulations amounts to $14.1 \%$ in the chamber. This somewhat larger difference may be related to the far more complex and highly unsteady hydrodynamics observed in the vortex chamber. Strongly time-depending and non-isotropic flow conditions are found there, challenging both experimental procedures and numerical modeling. The somewhat higher velocities found in the CFD could indicate that the shear stresses in the real environment are larger than the shear stresses predicted by the turbulence model, so that dissipation is too low. Overall, it is expected that CFD would tend to minimize a variety of practical losses, since they are not implemented in the simulation. Therefore, a slight overestimation of the measured velocities is not surprising. Considering the challenging hydrodynamics found close to the turbine 
(unsteady, free-surface turbulent flow with moving surfaces), the accuracy of the numerical predictions is still considered as satisfactory.
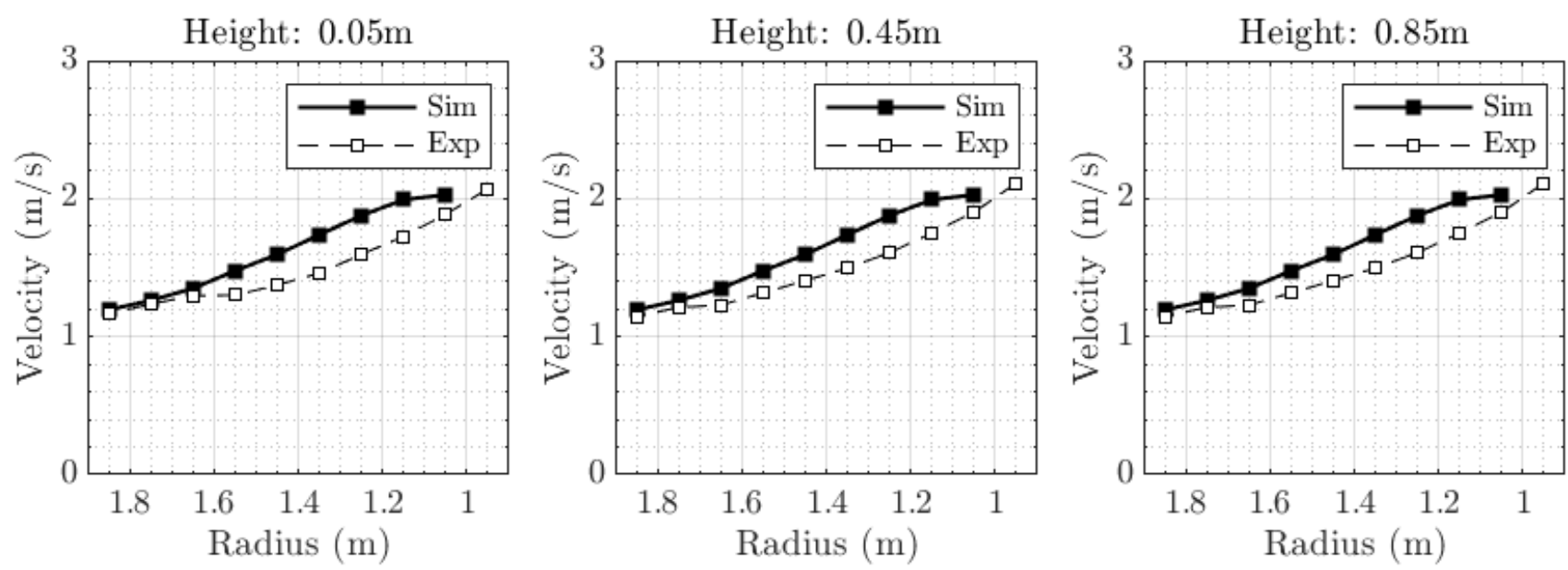

Figure 9. Comparison between simulation and experimental data within the vortex chamber for three selected heights above bottom. The outer wall of the chamber is situated at a radius of $1.95 \mathrm{~m}$ radius (on the right), while the turbine blades end at a radius of $0.875 \mathrm{~m}$ (on the left): (Left) height of $0.05 \mathrm{~m}$; (Middle) height of $0.45 \mathrm{~m}$; and (Right) height of $0.85 \mathrm{~m}$ above bottom in the vortex chamber. The average relative difference between numerical and experimental results is, from left to right: $10.7 \%, 14.7 \%$ and $12.5 \%$. The maximum relative differences are: $18.04 \%, 20.79 \%$ and $19.17 \%$.

Finally, time-averaged velocity fields were compared, to determine and locate the areas where the differences between simulation and experimental data are largest. In Figure 10, the corresponding fields of velocity magnitude are presented in the measurement section within the vortex chamber. Associated to this, Figure 11 shows the corresponding relative deviations, taking the experimental data as a reference.

Again, a general over-prediction is observed in CFD compared to experimental measurements. The relative deviations are highest in the central region of the vortex chamber. At least two reasons could be found for this. First, this is a flow region far from any wall and any guiding structure, so that large-scale turbulent vortices can freely develop in any direction and at a variety of scales, challenging turbulence modeling as well as accurate measurements. Additionally, this region corresponds in CFD to the transition from the rotating overset mesh (turbine region) to the static background mesh (rest of the geometry). The communication of flow information between the two meshes relies on interpolation, which could reduce the locally achieved accuracy. Reducing this issue would be possible by refining further the discretization in time and/or space, but at the cost of a much longer computational time. With a view toward parametric studies, the current model delivers an acceptable accuracy for guiding future improvements.

The computational fluid dynamics simulations agree well with experimental measurements. A sufficiently fine mesh was identified, involving roughly 4.7 million cells. Suitable boundary conditions were implemented. At the end, the obtained velocity fields match the experimental observations with a mean relative deviation of $7.5 \%$ at the chamber inlet and $14.1 \%$ within the vortex chamber itself. A better agreement was obtained regarding water level heights in the system, with relative differences not exceeding $4 \%$. Based on the results presented, the authors consider the numerical approach to be feasible for future investigations. The model is found to be a reliable and robust template for simulating large-scale water vortex power plants. Unfortunately, a direct comparison to other studies is not possible. Available studies with comparable approaches lack useful experimental data and therefore validation, as is the case for the work of Müller et al. [11]. In the case of Nishi and Inagaki [20], who provided both numerical and experimental data, the model was validated over the performance characteristics of the turbine, rather than the flow 
field itself. They also investigated a slightly different geometrical configuration of a WVPP, which makes a comparison even more difficult.

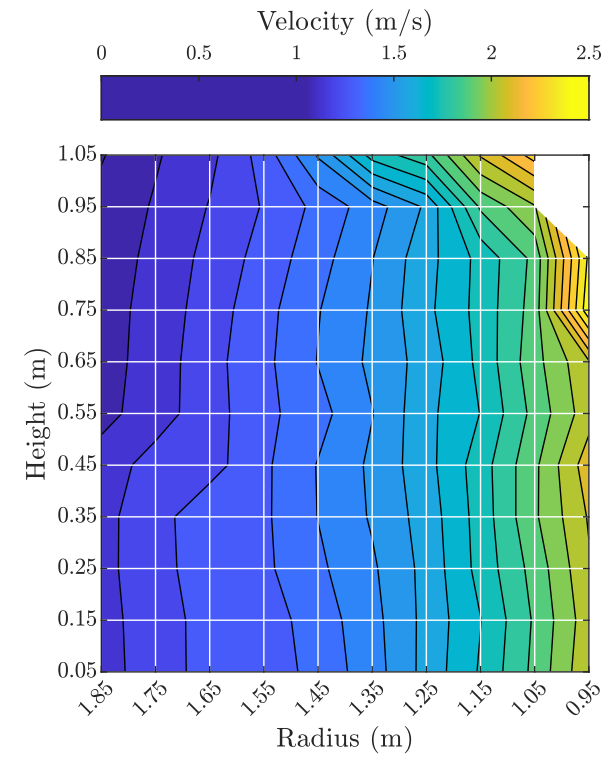

(a) Experimental data

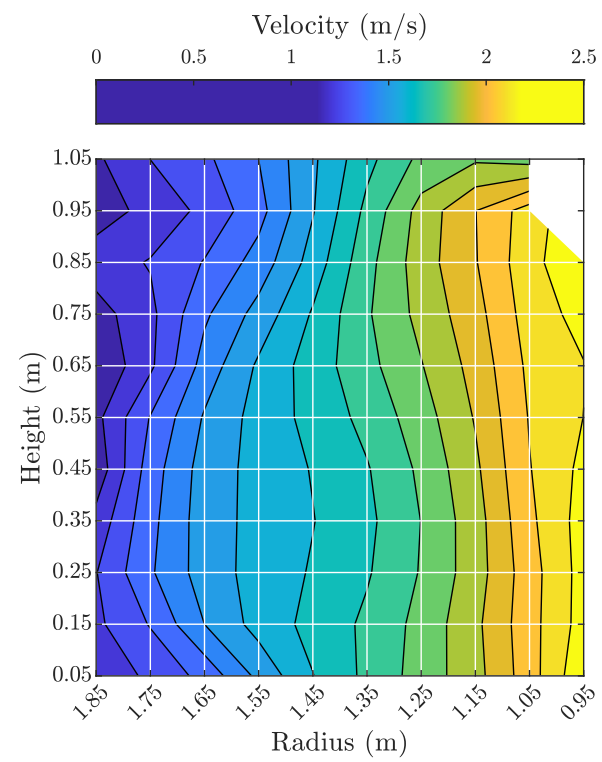

(b) Simulation data

Figure 10. Comparison of the fields of velocity magnitude in the vortex chamber, time-averaged over $10 \mathrm{~s}$ in the simulation and over $300 \mathrm{~s}$ in the experiment. The coordinates are the same as in Figure 9. The color scale is identical for both figures, and it has been shifted to focus more on the velocities from 1 to $2.5 \mathrm{~m} \mathrm{~s}^{-1}$ for a clearer comparison. Iso-velocity lines have been drawn in the left figures using bi-linear interpolation in-between the measurements points, which are located every $10 \mathrm{~cm}$ in both directions (at the intersections of the white lines).

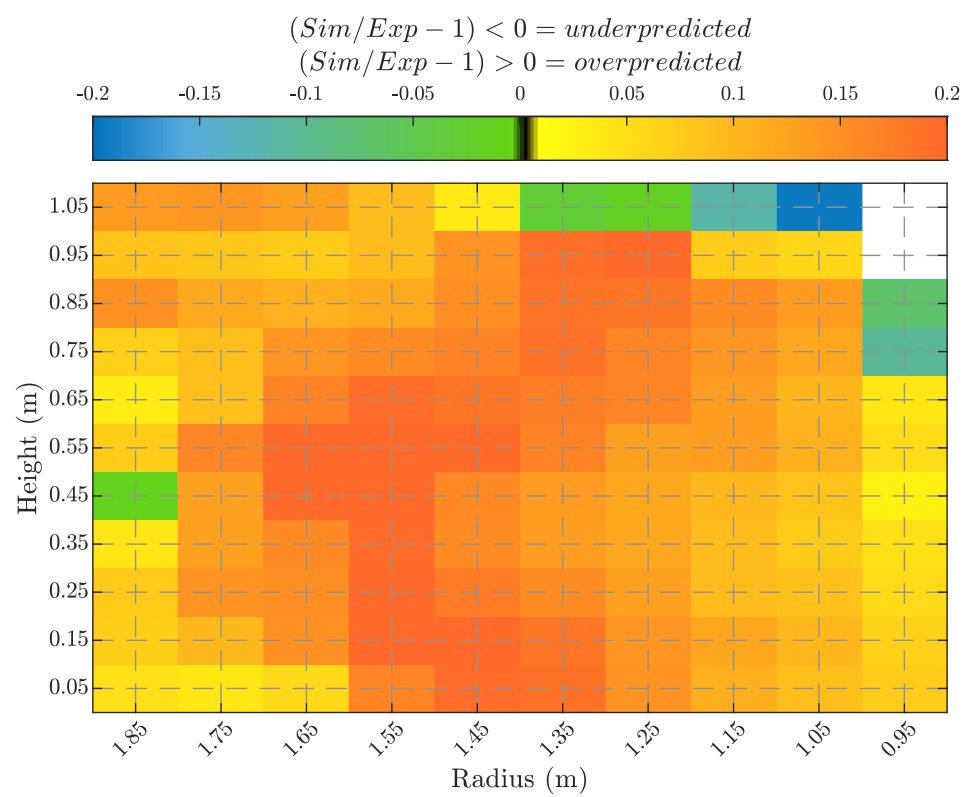

Figure 11. Relative deviation between the time-averaged velocities shown on both sides of Figure 10. The relative deviations are expressed as $\left(V_{\text {sim }} / V_{\text {exp }}\right)-1$, so that positive values indicate overprediction (yellow to orange), while negative values show under-prediction (green to blue).

\section{Conclusions}

Despite the challenging flow conditions (unsteady, highly turbulent flow with a free surface) and geometry (large installation with a full-scale rotating turbine but narrow gaps), a suitable numerical model for WVPP was developed. It involves solving the unsteady RANS equations in an Eulerian formulation combined with a VOF model to describe 
the free surface. Turbulence modeling relies on the the k- $\omega$-SST turbulence model. The persuasive accordance of numerics and experiments in both flow field and water levels for characteristic locations of the power plant make for a reliable numerical platform, with a meaningful trade-off between physical accuracy and numerical costs. Therefore, the model will allow for a deployment in subsequent studies as a digital twin of the facility as well as an ethohydraulic assessment of such a WVPP. Furthermore, a reliable prediction of the characteristic curves for a variety of inflow conditions and turbine rotations can be achieved.

Author Contributions: Conceptualization, D.P. and O.C.; methodology, D.P., O.C. and S.H.; experimental data, N.M.; writing—original draft preparation, D.P.; writing—review and editing, S.H., O.C. and D.T.; supervision, S.H.; and project administration and funding acquisition, S.H., D.T., N.M. and J.S. All authors have read and agreed to the published version of the manuscript.

Funding: This research was funded by the Bundesministerium für Bildung und Forschung (German Federal Ministry of Education and Research) as part of the project "VP4: Kaskade Fischfreundliches Wehr" 03WKC04.

Conflicts of Interest: The authors declare no conflict of interest.

\section{Abbreviations}

The following abbreviations are used in this manuscript:

$\begin{array}{ll}\text { ADV } & \text { Acoustic Doppler Velocimetry } \\ \text { CFD } & \text { Computational Fluid Dynamics } \\ \text { CPU } & \text { Central Processing Unit } \\ \text { Exp } & \text { Experiment } \\ \text { FFW } & \text { Fish-Friendly-Weir } \\ \text { k } & \text { turbulent kinetic energy } \\ \text { LES } & \text { Large-Eddy Simulations } \\ \text { RANS } & \text { Reynolds-Averaged Navier-Stokes } \\ \text { Sim } & \text { Simulation } \\ \text { SIMPLE } & \text { Semi-implicit Method for Pressure Linked Equations } \\ \text { SST } & \text { Shear Stress Transport } \\ \text { TUD } & \text { Technical University of Dresden } \\ \text { VOF } & \text { Volume of Fluid } \\ V_{\text {exp }} & \text { averaged velocity value of experimental data } \\ V_{\text {sim }} & \text { averaged velocity value of simulation data } \\ \text { WVPP } & \text { water vortex power plant } \\ y^{+} & \text {non-dimensional wall distance } \\ \omega & \text { specific dissipation rate }\end{array}$

\section{References}

1. Bostan, I.; Gheorghe, A.V.; Dulgheru, V.; Sobor, I.; Bostan, V.; Sochirean, A. Resilient Energy Systems: Renewables: Wind, Solar, Hydro; Springer Science \& Business Media: Berlin, Germany, 2012; Volume 19.

2. Wagner, H.J.; Mathur, J. Introduction to Hydro Energy Systems: Basics, Technology and Operation; Springer Science \& Business Media: Berlin, Germany, 2011.

3. Anyi, M.; Kirke, B. Evaluation of small axial flow hydrokinetic turbines for remote communities. Energy Sustain. Dev. 2010, 14, 110-116. [CrossRef]

4. Silva, A.T.; Lucas, M.C.; Castro-Santos, T.; Katopodis, C.; Baumgartner, L.J.; Thiem, J.D.; Aarestrup, K.; Pompeu, P.S.; O’Brien, G.C.; Braun, D.C.; et al. The future of fish passage science, engineering, and practice. Fish Fish. 2018, 19, 340-362. [CrossRef]

5. EP. Directive 2000/60/EC of the European Parliament and of the Council of 23 October 2000 Establishing a Framework for Community Action in the Field of Water Policy; EP: Brussels, Belgium, 2000.

6. DWA. Fischaufstiegsanlagen und Fischpassierbare Bauwerke: Gestaltung, Bemessung, Qualitätssicherung, mai 2014 ed.; Number M 509 in DWA-Regelwerk; DWA: Hennef, Germany, 2014.

7. Giesecke, J.; Heimerl, S.; Mosonyi, E. Wasserkraftanlagen: Planung, Bau und Betrieb, 6th ed.; Springer: Berlin, Germany, 2014; OCLC: 879592116. [CrossRef]

8. Larsson, A. The Efficiency of a Fish Ladder for Salmonid Upstream Migration in a Swedish Stream. Master's Thesis, University of Gothenburg, Department of Biological and Environmental Sciences, Gothenburg, Sweden, 2016. 
9. Bunt, C.M.; Castro-Santos, T.R.; Haro, A. Reinforcement and validation of the analyses and conclusions related to fishway evaluation data from Bunt et al.:'Performance of fish passage structures at upstream barriers to migration'. River Res. Appl. 2016, 32, 2125-2137. [CrossRef]

10. McLaughlin, R.L.; Smyth, E.R.B.; Castro-Santos, T.; Jones, M.L.; Koops, M.A.; Pratt, T.C.; Vélez-Espino, L.A. Unintended consequences and trade-offs of fish passage. Fish Fish. 2013, 14, 580-604. [CrossRef]

11. Müller, S.; Cleynen, O.; Hoerner, S.; Lichtenberg, N.; Thévenin, D. Numerical analysis of the compromise between power output and fish-friendliness in a vortex power plant. J. Ecohydraulics 2018, 3, 86-98. [CrossRef]

12. Lichtenberg, N.; Cleynen, O.; Thévenin, D. Numerical investigations of a water vortex hydropower plant implemented as fish ladder. In Proceedings of the 4th IAHR Europe Congress, Liege, Belgium, 27-29 July 2016; p. 277.

13. Kanemoto, T.; Inagaki, A.; Misumi, H.; Kinoshita, H. Development of gyro-type hydraulic turbine suitable for shallow stream (1st report, rotor works and hydroelectric power generation). Trans. Jpn. Soc. Mech. Eng. Ser. B 2004, 70, 413-418. [CrossRef]

14. Dhakal, S.; Nakarmi, S.; Pun, P.; Thapa, A.B.; Bajracharya, T.R. Development and testing of runner and conical basin for gravitational water vortex power plant. J. Inst. Eng. 2014, 10, 140-148. [CrossRef]

15. Dhakal, S.; Timilsina, A.B.; Dhakal, R.; Fuyal, D.; Bajracharya, T.R.; Pandit, H.P.; Amatya, N.; Nakarmi, A.M. Comparison of cylindrical and conical basins with optimum position of runner: Gravitational water vortex power plant. Renew. Sustain. Energy Rev. 2015, 48, 662-669. [CrossRef]

16. Wanchat, S.; Suntivarakorn, R.; Wanchat, S.; Tonmit, K.; Kayanyiem, P. A parametric study of a gravitation vortex power plant. Adv. Mater. Res. 2013, 805, 811-817. [CrossRef]

17. Müller, N.; Stamm, J.; Wagner, F. A water vortex power plant as ethohydraulic test site. In Proceedings of the CongressInternational Association for Hydraulic Research (IAHR), Trento, Italy, 12-14 June 2018. [CrossRef]

18. Wagner, F.; Warth, P.; Royan, M.; Lindig, A.; Müller, N.; Stamm, J. Laboruntersuchungen zum Fischabstieg über ein Wasserwirbelkraftwerk. Wasserwirtschaft 2019, 109, 64-67. [CrossRef]

19. Mulligan, S.; Casserly, J.; Sherlock, R. Experimental and numerical modelling of free-surface turbulent flows in full air-core water vortices. In Advances in Hydroinformatics; Springer: Berlin/Heidelberg, Germany, 2016; pp. 549-569.

20. Nishi, Y.; Inagaki, T. Performance and flow field of a gravitation vortex type water turbine. Int. J. Rotating Mach. 2017, 2017, 2610508. [CrossRef]

21. Dhakal, R.; Bajracharya, T.; Shakya, S.; Kumal, B.; Khanal, K.; Williamson, S.; Gautam, S.; Ghale, D. Computational and experimental investigation of runner for gravitational water vortex power plant. In Proceedings of the 2017 IEEE 6th International Conference on Renewable Energy Research and Applications (ICRERA), San Diego, CA, USA, 5-8 November 2017; pp. 365-373.

22. Sedai, A.; Yadav, B.K.; Kumal, B.B.; Khatiwada, A.; Dhakal, R. Performance analysis of Gravitational water vortex power plant using scale-down model. In Proceedings of the Current Research in Hydropower Technologies (CRHT X), Dhulikhel, Nepal, 28 April 2020.

23. Bajracharya, T.R.; Shakya, S.R.; Timilsina, A.B.; Dhakal, J.; Neupane, S.; Gautam, A.; Sapkota, A. Effects of Geometrical Parameters in Gravitational Water Vortex Turbines with Conical Basin. J. Renew. Energy 2020, 2020, 5373784.

24. Hirt, C.W.; Nichols, B.D. Volume of fluid (VOF) method for the dynamics of free boundaries. J. Comput. Phys. 1981, 39, 201-225. [CrossRef]

25. Menter, F.R. Two-equation eddy-viscosity turbulence models for engineering applications. AIAA J. 1994, 32, 1598-1605. [CrossRef]

26. Wallin, S.; Johannsen, A.V. An explicit algebraic Reynolds stress model for incompressible and compressible turbulent flows. $J$. Fluid Mech. 2000, 403, 89-132. [CrossRef]

27. Müller, N.; Jähnel, C.; Stamm, J.; Wagner, F. Analyse der Strömung in einem Wasserwirbelkraftwerk hinsichtlich des Fischabstiegs. Wasserwirtschaft 2019, 109, 60-63. [CrossRef]

28. Müller, N.; Stamm, J. Errichtung eines 1:1 Labormodells für ethohydraulische Untersuchungen an einem Wasserwirbelkraftwerk. In Dresdner Wasserbauliche Mitteilungen (Heft 60); Technische Universität Dresden, Institut für Wasserbau und technische Hydromechanik: Dresden, Germany, 2018; pp. 123-132. 